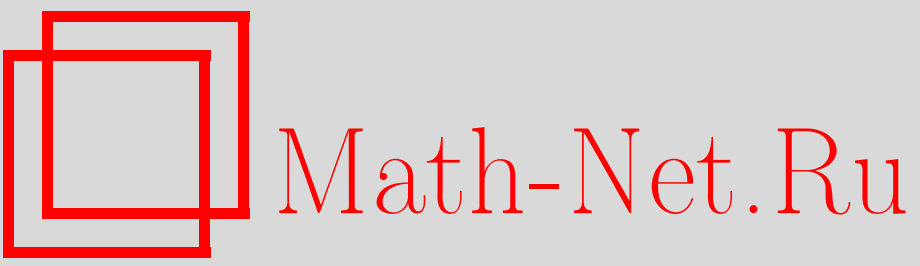

А. Г. Нурмиев, Замыкания нильпотентных орбит кубических матриц порядка три, УМH, 2000, том 55, выпуск 2, 143-144

DOI: https://doi.org/10.4213/rm279

Использование Общероссийского математического портала Math-Net.Ru подразумевает, что вы прочитали и согласны с пользовательским соглашением

http://www.mathnet.ru/rus/agreement

Параметры загрузки:

IP: 18.234 .197 .8

26 апреля 2023 г., 14:49:22 


\title{
ЗАМЫКАНИЯ НИЛЬПОТЕНТНЫХ ОРБИТ КУБИЧЕСКИХ МАТРИЦ ПОРЯДКА ТРИ
}

\author{
А. Г. НурмиЕВ
}

1. В заметке описывается структура замыканий нильпотентных орбит естественного представления группы $G=S L\left(V_{1}\right) \times S L\left(V_{2}\right) \times S L\left(V_{3}\right)$ в пространстве $U=V_{1} \otimes V_{2} \otimes V_{3}$, где $V_{i}$ - трехмерное комплексное векторное пространство, $i=1,2,3$. Пусть $\left\{e_{1}, e_{2}, e_{3}\right\}$ - базис в $V_{1}$, $\left\{e_{4}, e_{5}, e_{6}\right\}-$ в $V_{2},\left\{e_{7}, e_{8}, e_{9}\right\}-$ в $V_{3}$.

Элемент из $U$ называется нилпотентным, если замыкание его орбиты содержит нуль. Орбита, содержащая нильпотентный элемент, называется нильпотентной. Описание нильпотентных орбит рассматриваемого представления получается из [1; табл. 5] путем разнесения каждого представителя при помощи группы $T$, циклически переставляющей $V_{1}, V_{2}, V_{3}$. Следовательно, мы получаем из каждой орбиты в [1] одну или три орбиты для нашего случая. Очевидно, что достаточно знать замькания орбит представителей, указанных в [1].

\begin{tabular}{|r|l|r|l|}
\hline № & Представитель & $\operatorname{dim} S$ & Номера орбит в замыкании \\
\hline 1 & 159168249258267347 & 0 & $2,2^{\prime \prime}, \geqslant 3$ \\
2 & 159168249257258347 & 1 & $3,3^{\prime}, 3^{\prime \prime}, 4^{\prime}, 4^{\prime \prime}, \geqslant 5$ \\
3 & 149158167248259347 & 2 & $5^{\prime}, 5^{\prime \prime}, 6, \geqslant 7 \backslash 9^{\prime \prime}$ \\
4 & 149158248257367 & 2 & $5,6,7,7^{\prime}, 8,9^{\prime \prime}, \geqslant 10 \backslash 11^{\prime \prime}$ \\
5 & 149167168257348 & 3 & $7,7^{\prime}, 8,9^{\prime \prime}, \geqslant 10 \backslash 11^{\prime \prime}$ \\
6 & 149158248267357 & 3 & $7,7^{\prime}, \geqslant 10 \backslash 11^{\prime \prime}$ \\
7 & 149158167248357 & 4 & $10,11,12^{\prime}, 12^{\prime \prime}, \geqslant 13 \backslash 15$ \\
8 & 149167258347 & 4 & $\geqslant 10 \backslash 11,11^{\prime}, 11^{\prime \prime}$ \\
9 & 147158258269 & 4 & $12,13,15,16,17,18,18^{\prime \prime}, 19,19^{\prime \prime}, 20,21,22$, \\
& & & $23,23^{\prime}, 23^{\prime \prime}, 24,25$ \\
10 & 149158167248257347 & 5 & $\geqslant 13 \backslash 15,15^{\prime}, 15^{\prime \prime}$ \\
11 & 149167248357 & 5 & $14^{\prime}, 15^{\prime}, 15^{\prime \prime}, 16^{\prime}, 16^{\prime \prime}, 17,19,19^{\prime}, 19^{\prime \prime}, 20,21$, \\
& & & $22^{\prime}, 22^{\prime \prime}, 23,23^{\prime}, 23^{\prime \prime}, 24,25$ \\
12 & 149167247258 & 5 & $13,15,16,17,18,18^{\prime \prime}, 19,19^{\prime \prime}, 20,21,22,23$, \\
& & & $23^{\prime}, 23^{\prime \prime}, 24,25$ \\
13 & 149158167248257 & 6 & $16,17,18,18^{\prime \prime}, 19,19^{\prime \prime}, 20,21,22,23,23^{\prime}$, \\
& & & $23^{\prime \prime}, 24,25$ \\
14 & 149157168247348 & 7 & $16,16^{\prime}, 17^{\prime \prime}, 19,19^{\prime}, 19^{\prime \prime}, 20,21,22,22^{\prime}, 23$, \\
& & & $23^{\prime}, 23^{\prime \prime}, 24,25$ \\
15 & 158169247 & 7 & $16,19,19^{\prime \prime}, 20,21,22,23,23^{\prime}, 23^{\prime \prime}, 24,25$ \\
16 & 149158167247 & 8 & $19,19^{\prime \prime}, 20,21,22,23,23^{\prime}, 23^{\prime \prime}, 24,25$ \\
17 & 148157249267 & 8 & $19,19^{\prime \prime}, 20,21,23,23^{\prime}, 23^{\prime \prime}, 24,25$ \\
18 & 147158248259 & 8 & $19,20,21,23,23^{\prime}, 23^{\prime \prime}, 24,25$ \\
19 & 149157248 & 9 & $20,21,23,23^{\prime}, 23^{\prime \prime}, 24,25$ \\
20 & 147258 & 10 & $21,23,23^{\prime}, 23^{\prime \prime}, 24,25$ \\
21 & 148157247 & 11 & $23,23^{\prime}, 23^{\prime \prime}, 24,25$ \\
22 & 147158169 & 13 & $23,24,25$ \\
23 & 147158 & 14 & 24,25 \\
24 & 147 & 24 & 25 \\
25 & ноль & 24 & \\
\hline
\end{tabular}

Работа выполнена при поддержке Российского фонда фундаментальных исследований (грант № 98-01-00598). 
2. Для описания замыканий нильпотентных орбит воспользуемся методом, изложенным в [2; пп. 5.5, 5.6]. Согласно [3], рассматриваемое представление является представлением $\theta$-группы (см. [3; № 2 в табл.]). В [4; $\S 2]$ было дано определение характеристики нильпотентного элемента для представлений $\theta$-групп. Рассуждая аналогично [2; п. 5.5, пример 3], можно показать, что данное определение совпадает с определением характеристики, данным в [2; п. 5.5]. Заметим, что характеристики нильпотентных элементов можно получить из [1; табл. 5]. Поскольку характеристики неэквивалентных нильпотентных элементов не сопряжены (см. [4; п. 2]), то страты, введенные в $[2 ;$ п. 5.6], совпадают с орбитами. Следовательно, замыкание орбиты совпадает с замыканием страта, а оно имеет простой вид.

А именно, пусть $e \in U$ - нильпотентный элемент, $h_{e}$ - его характеристика, лежащая в касательной алгебре группы $G$. Рассмотрим разложение $U$ на собственные подпространства $V_{\lambda}\left(h_{e}\right)$ относительно $h_{e}$. Положим, $V_{+}\left(h_{e}\right)=\bigoplus_{\lambda \geqslant 2} V_{\lambda}\left(h_{e}\right)$. Тогда замыкание страта, содержащего $e$, а следовательно, и орбиты точки $е$, есть $G V_{+}\left(h_{e}\right)$.

3. Пусть $e_{1}, e_{2} \in U-$ нильпотентные элементы. Из вышесказанного следует, что условие $G e_{1} \subset \overline{G e_{2}}$ равносильно тому, что $G e_{1}$ пересекает $V_{+}\left(h_{e_{2}}\right)$. Проверяя это условие и используя соображения размерности, получаем приведенную здесь таблицу. В первой графе указан номер орбиты, в третьей - размерность стабилизатора $S$ (см. [1]). Вектор $e_{i} \otimes e_{j} \otimes e_{k}$ обозначается через $i j k, e_{i} \otimes e_{j} \otimes e_{k}+e_{p} \otimes e_{q} \otimes e_{r}-$ через $i j k p q r$, и так далее для любого числа членов. "Представитель" - элемент из соответствующей орбиты. Пусть $t_{0} \in T$ отвечает перестановке $(1,2,3)$. Для любого $n=1, \ldots, 25 n, n^{\prime}, n^{\prime \prime}$ в четвертой графе означает номер орбиты элемента, полученного из $n$-го элемента второй графы при помощи id, $t_{0}, t_{0}^{2}$ соответственно (см. п. 1$)$. Символ " $\geqslant k$ " означает "все орбиты с номерами $i, i^{\prime}, i^{\prime \prime}$, где $i \geqslant k$ ", а " $k_{1}, k_{2} \ldots$ " - "за исключением орбит с номерами $k_{1}, k_{2} \ldots$..

Автор благодарен своему научному руководителю Э. Б. Винбергу за постановку задачи и постоянное внимание к работе.

\section{СПИСОК ЛИТЕРАТУРЫ}

[1] Винберг Э. Б., Элашвили А. Г. // Тр. семинара по вект. тенз. анализу. Т. 18. М.: Изд-во МГУ, 1978. С. 197-233. [2] Винберг Э. Б., Попов В. Л. // Итоги науки и техн. Соврем. пробл. матем. Фунд. направления. Т. 55. М.: ВИНИТИ, 1989. С. 137-311. [3] Винберг Э. Б. // Изв. АН СССР Сер. матем. 1976. Т. 40. №3. С. 488-526. [4] Винберг Э. Б. // Тр. семинара по вект. тенз. анализу. Т. 19. М.: Изд-во МГУ, 1979. С. 155-177.

Московский государственный

Принято редколлегией университет им. М. В. Ломоносова 26.01.2000 